\title{
5th Annual Quality Healthcare Information on the 'Net Conference Access, Quality, and Patient Empowerment
}

\section{Internet Healthcare Coalition}

\section{Online peer support groups in mental health}

Robert Hsiung

Associate Professor of Clinical Psychiatry at the University of Chicago, USA

In online peer support groups, patients from different areas within a country -- or even from different countries -- work together to support and educate each other. Some groups are facilitated by mental health professionals, others by laypeople, others not at all.

In this session, three facilitated online peer support groups in mental health are examined. The different groups are visited, and samples of interactions at each are presented. The groups are then systematically compared in terms of activity level (overall, by topic, and by user), types of communication (questions vs. information vs. opinions vs. suggestions), and technical features (for example, the ability to search archives). Finally, group policies (regarding, for example, what types of messages are allowed and how the privacy of users is protected) are contrasted.

\section{Empowering consumers and physicians}

Lone Hummelshoj

Editor of EndoZone.org and Chair of Endometriose Foreningen Denmark

The educated consumer has arrived. She will increasingly do her own research and present this to her physician before discussing individual treatment options. But who should be providing this information? Physicians or lay-organisations?

In her presentation, Lone Hummelshoj will present strategies to disseminate factual information to empower consumers whilst keeping physicians abreast of what their patients are reading. This session will also discuss how to encourage physicians to participate in web-based specialty forums, in order to gain the most from the plethora of information available on the internet.

\section{The Empowered Patient - Online answers to specific and pertinent information in an evidence} based context

\author{
Ahmad Risk and Michael Miller \\ $U K$
}

This presentation describes the 'Empowered Patient' website, and the concepts behind its creation. 
Seekers of health information require that information empower them to make better health and medical care choices; be assured the information is current, accurate and backed by evidence whenever possible; and most importantly, arrive at the right information quickly and easily at the right time.

We believe that validated, independent and impartial medical information, delivered in a quick and straightforward manner and in appropriate language is paramount in deploying the internet in providing the public with just in time high quality information.

The primary objectives of the 'Empowered Patient' website are to answer these 3 questions:

1. Which procedure?

By reference to options, their risks, meaning of terms and specialties.

2. Which doctor?

Finding the consultants or surgeons best suited for a specific medical condition and personal circumstances and preferences.

3. Which hospital?

By reference to where would be the best place to have that particular procedure done, and what is the best value in terms of cost and back up and support facilities.

Notwithstanding the above ordering, the site works on the principle that its first task is to help patients be seen quickly by the most appropriate medical specialist for their specific conditions on the basis of their subspecialties and special interests. The choice of the most appropriate procedure and hospital setting is seen to flow from the initial right choice of medical specialist.

In addition, the site provides other valuable information, including:

- How to get the best medical care

- $\quad$ Disease-specific questions to ask before treatment

- Treatment options

- How to choose a hospital

- $\quad$ Treatment pricing data

- Database of validated providers

\section{Concerning the Internet: What are patients' needs?}

Arjan Meutgeert and Johan Beun, $N P C F$, The Netherlands

- $\quad$ Patients deserve respect;

- $\quad$ Patients should empower themselves;

- Patients need quality information;

- Patients need protection.

Respect: Patients' decisions about their own life need to be respected. They have needs, perspectives and preferences that should be taken into account, and respected, by those whose role it is to help. This is in accordance with a patient-centered approach to health care. Accordingly, the patientdoctor relationship will be altered from "do what the doctor tells you" towards a collaborative relationship between equals. They are both experts: the doctor in the medical field, the patient in his life. Together they can merge their knowledge and needs in ways which enable the patient to make balanced decisions in his or her life. 
Empowerment: Patients need to empower themselves in many ways. On the personal level they should be informed on issues relevant to their health, as well as their rights and responsibilities. On the organizational level, patient advocacy groups and national patients organizations should have sufficient financial support to organize themselves in a professional way. They also need to be included as active, equal partners in policy making and in bilateral talks with governments, insurers and health professionals.

Patients need quality communication, information and transparency: As patients seek to be informed, they will need to assess whether or not the information they access is reliable, trustful, understandable and educating. Support and guidelines for navigating though the multitude of information sources available on the net should be easily accessible. This includes communication with other patients, governments, health-providers, etc., as well as trustful accreditation systems.

Patients need data protection: Patients need guarantees that their (medical) data is protected and that nobody can access it against their will. Furthermore, they need to understand how their own net-behavior can compromise privacy, along with ways to protect themselves. 